\title{
The influence of convective energy transport on dust formation in brown dwarf atmospheres
}

\author{
Matthias Dehn ${ }^{1}$, Christiane Helling ${ }^{2}$, \\ Peter Woitke $^{3}$ and Peter Hauschildt ${ }^{1}$ \\ ${ }^{1}$ Hamburger Sternwarte, Universität Hamburg, Gojenbergsweg 112, 21029 Hamburg, Germany \\ email: mdehn@hs.uni-hamburg.de \\ ${ }^{2}$ Research and Scientific Support Department ESTEC/ESA, PO Box 299, 2200 AG Noordwijk, \\ The Netherlands \\ ${ }^{3}$ Sterrewacht Leiden, PO Box 9513, 2300 RA Leiden, The Netherlands
}

\section{Introduction}

Convection is the main energy transport mechanism in brown dwarf atmospheres. The overshoot of ascending bubbles affects the upper and cooler region where dust formation can occur. In order to understand the observed presence of dust, the formation of grains and their gravitational settling must be balanced by up-mixing of condensible elements via convective overshoot. Otherwise, brown dwarf atmospheres would be completely dustfree (Woitke \& Helling 2004).

The process of dust formation in models of brown dwarf atmospheres has been treated only in phase equilibrium models so far. This allowed to take into account many solid and liquid species and their feedback onto the atmosphere's element abundances and the radiation field (e.g., Allard et al. 2001). However, the actual formation process could not be treated. On the other hand, models which treat the dust formation in more detail by, e.g., a kinetic approach, cannot yet take into account the feedback onto the atmospheric structure (Woitke \& Helling 2004, Helling et al. 2006).

We present results of the coupling between the classical stellar atmosphere problem and a kinetic theory of oxygen-rich dust formation and gravitational settling. This allows us to study, for instance, the feedback between dust formation and convection. The results are represented in the frame of a consistent stellar atmosphere simulation applying standard mixing length theory.

\section{Approach}

We solve the classic stellar atmosphere problem with PHOENIX (Hauschildt \& Baron 1999), by calculating the frequency dependent radiative transfer for gas and dust, and the structure of the atmosphere in hydrostatic equilibrium. The atmospheric structure $(T, \rho)$ and the convective velocity are the input for the quasi static dust-model (Woitke \& Helling 2003) which treats the nucleation of $\mathrm{TiO}_{2}$-seed particles, the dirty growth and evaporation of the dust grains $\left(\mathrm{Fe}[\mathrm{s}], \mathrm{SiO}_{2}[\mathrm{~s}], \mathrm{Mg}_{2} \mathrm{SiO}_{4}[\mathrm{~s}], \mathrm{TiO}_{2}[\mathrm{~s}], \mathrm{Al}_{2} \mathrm{O}_{3}[\mathrm{~s}]\right.$ ), and gravitational settling. The dust module provides, e.g., the mean particle size $\langle a\rangle$ as function of atmospheric height. The element abundances are the result of element consumption by dust formation.

The opacities of the dust grains are calculated using a Mie theory code (Wolf \& Voshchinnikov 2004). We assume silicate refractive indices. The model parameters are 
characteristic for early L-type brown dwarfs: $\mathrm{T}_{\text {eff }}=2300 \mathrm{~K}, \log g=5.0$, and solar abundances (Grevesse et al. 1993).

In order to examine the influence of the convective flux on the nucleation rate of dust seeds and the mean grain size, we vary the mixing length parameter $l / H_{\mathrm{p}}=1.0$, 2.0 (standard), 4.0. The depth dependent mixing time scale $\tau_{\text {mix }}$ is needed for the dust moment equations to be solved. In a simplified treatment of the mixing, $1 / \tau_{\text {mix }}$ scales logarithmic linear with a slope of the overshoot parameter $\beta=2.2$ outside the convection zone according to Ludwig et al. (2002).

\section{Results}

The convective speed is a result of mixing length theory in PHOENIX (Fig. 1). The mixing time scale $\tau_{\text {mix }}$ is shown in the atmosphere layers where dust is present (Fig. 2). It exists also inside the convection zone with $\tau_{m i x}=l / v_{\text {conv }}$ where it has no effect on dust formation. Minimal mixing time scales lie between $129 \ldots 310 \mathrm{~s}$ at about 1 bar.

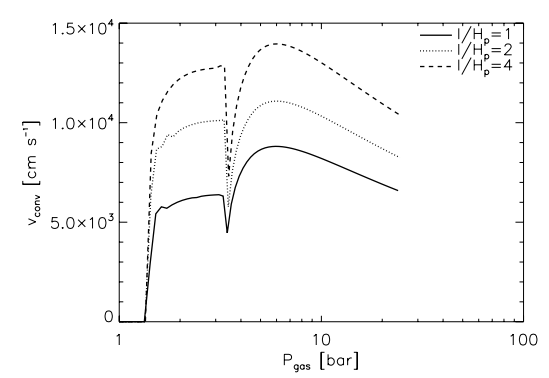

Figure 1. Convective speed $v_{\text {conv }}$

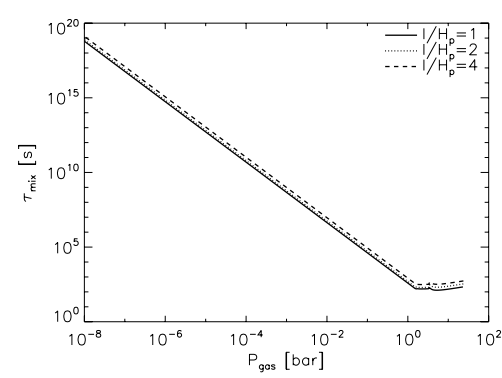

Figure 2. Convective mixing time scale $\tau_{\text {mix }}$

The nucleation rate $J^{*}$ is strongly peaking in a very narrow range of the atmosphere (Fig. 3). With higher mixing time scales at $l / H_{\mathrm{p}}=4, J^{*}$ drops by a factor of $1 / 3$ compared to models with lower $l / H_{\mathrm{p}}$ (Fig. 3). Calculations in Woitke \& Helling (2004, Fig. 4) show a significant change of the mean dust grain size $\langle a\rangle$ by changing the mixing time scale. In our calculations the minimal mixing time scale remains in the same order of magnitude, which is different to the study presented by Woitke \& Helling (2004). The mean grain size (Fig. 4) is negligibly affected by a changing mixing efficiency in our relatively hot L-dwarf model in which the size of the dust grains remains generally small.

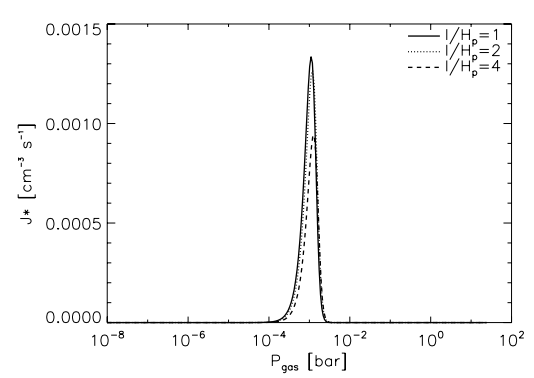

Figure 3. Nucleation rate $J_{*}$

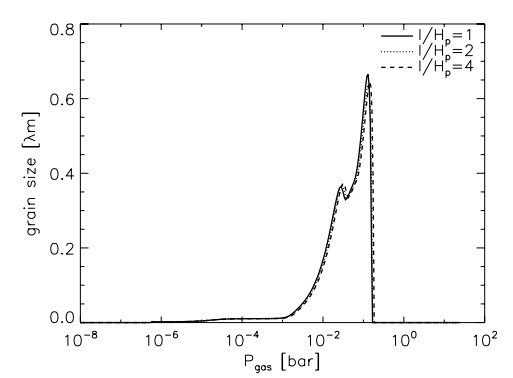

Figure 4. Mean grain size $\langle a\rangle$ 
As depicted in figure 5 and 6 , the number density of dust particles and the dust-to-gas density ratio change with varying $l / H_{\mathrm{p}}$ by about the same order as the nucleation rate changes. The chemical composition of the dust seems to be unaffected by changing mixing efficiencies, because the amount of condensible material available is mainly influenced by the mixing efficiency and not by the local temperature.

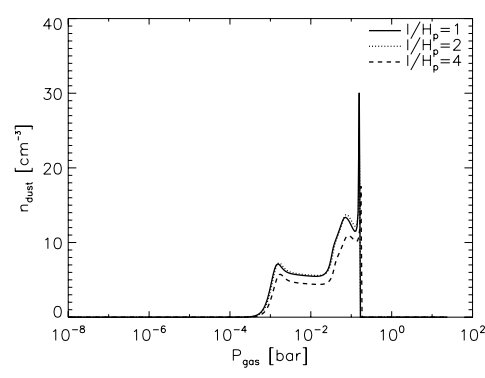

Figure 5. Number density of dust particles

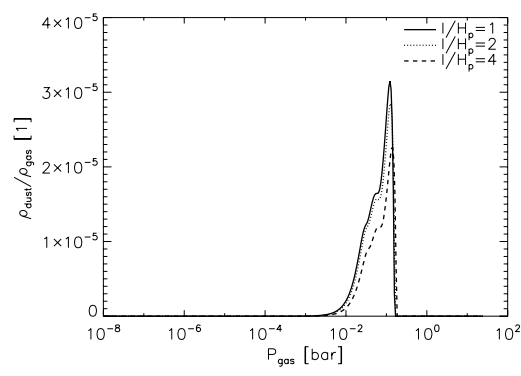

Figure 6. Dust-to-gas-mass density ratio

Generally, the amount of dust and the mean grain size (Fig. 4) remains small in the early L-type model atmosphere under investigation. Because of the dependence of the number density of dust particles on the mixing length at this effective temperature we expect a change of the strength of spectral dust features in the infrared.

\section{Outlook}

Brown dwarfs of later spectral types and respectively cooler effective temperatures $\left(\mathrm{T}_{\text {eff }}=2000 \ldots 1000 \mathrm{~K}\right)$ produce more dust in their atmosphere which influences the radiation field and its spectral appearance much stronger. However, the study of the early-type brown dwarfs, as shown in this poster, does allow the understanding of the onset of dust formation during the brown dwarf's evolution.

Future work should examine, if a change of the mixing length makes a difference to the dust complex including information on, e.g., mean grain size and dust composition at lower effective temperatures.

\section{References}

Allard, F., Hauschildt, P.H., Alexander, D.R., Tamanai, A. \& Schweitzer, A. 2001, ApJ 556, 357

Grevesse, N., Noels, A. \& Sauval, A.J. 1993, A\&A 271, 587

Hauschildt, P.H. \& Baron, E. 1999, J. Comput. Appl. Math. 109, 41

Helling, Ch., Thi, W.-F., Woitke, P., \& Fridlund, M. 2006, A\&A 451, L9

Ludwig H.-G., Allard, F. \& Hauschildt, P.H. 2002, A\&A 395, 99

Woitke, P., Helling, Ch. 2003, A\&A 399, 297

Woitke, P., Helling, Ch. 2004, A\&A 414, 335

Wolf, S. \& Voshchinnikov, N.V. 2004, Comp. Phys. Comm. 162, 113 